\title{
Interventions to Improve HIV Viral Load Suppression among the Adolescents: Evidence of Improvement Science through a Quality Improvement Approach in Eastern Uganda
}

\author{
Bonniface Oryokot, Yunus Miya, Barbara Logose, Eunice Ajambo, Abraham Ignatius Oluka, \\ Charles Odoi, Bernard Michael Etukoit, Levicatus Mugenyi, Kenneth Mugisha \\ Department of Program Management and Capacity Development, The AIDS Support Organization (TASO), \\ Kampala, Uganda \\ Email:bonory@gmail.com
}

How to cite this paper: Oryokot, B., Miya, Y., Logose, B., Ajambo, E., Oluka, A.I., Odoi, C., Etukoit, B.M., Mugenyi, L. and Mugisha, K. (2020) Interventions to Improve HIV Viral Load Suppression among the Adolescents: Evidence of Improvement Science through a Quality Improvement Approach in Eastern Uganda. World Journal of AIDS, 10, 94-106.

https://doi.org/10.4236/wja.2020.102008

Received: March 4, 2020

Accepted: May 8, 2020

Published: May 11, 2020

Copyright $\odot 2020$ by author(s) and Scientific Research Publishing Inc. This work is licensed under the Creative Commons Attribution International License (CC BY 4.0).

http://creativecommons.org/licenses/by/4.0/ (c) (i) Open Access

\begin{abstract}
Introduction: Achieving viral load suppression among the adolescents living with HIV continues to hold back attainment of sustainable development goals. TASO Mbale realized a viral load suppression rate of $63.1 \%$ among the adolescents living with HIV in care in quarter 4 of 2016. We therefore, instituted a quality imrpovement project to improve Viral load suppression from $63.1 \%$ in quarter 42016 to $90 \%$ by the end of quarter 4 2017. Method: Baseline data from the Uganda viral load dashboard were analyzed, and fishbone diagram was utilized to provide root causes of low viral load suppression among the adolescents living with HIV at TASO Mbale. The identified barriers were Knowlegde gap, among the adolescents, on positive living, Missing clinic appointments, Sub-optimal adherence, Poorly planned adolescent HIV clinic, Inadequate follow-up and Low use of data for informed decisions. A plan-do-study-act (PDSA) model was applied to implement tested changes. Strategies that worked included introduction of appointment register to track appointment behaviour of the adolescents, generating lists of clients on appointment who were due for Viral Load bleeding, telephone calls for follow up, increasing the frequency of reviewing adolescents from once a month to twice a week, committing a dedicated team responsible for adolescent care. Results: The viral load suppression improved from $63.1 \%$ in quarter 4 of 2016 to $63.8 \%$ in the first quarter of 2017 , to $87.5 \%$ in quarter 2 of $2017,97.6 \%$ in the third quarter and $91.4 \%$ in quarter 4 of 2017. Conclusion: The use of quality improvement in addressing gaps in HIV service delivery is highly effective.
\end{abstract}


Keywords

HIV, Adolescents, Viral Load Suppression, Quality Improvement

\section{Introduction}

By 2018, about 74.9 million people have been infected by the Human Immune Deficiency Virus (HIV) with 32 million deaths globally since the epidemic was first identified [1]. The East and Southern Africa host up to 20.6 million persons living with HIV (PLHIV) and had about 800,000 new infections in 2018 [1]. This picture demonstrates how far the world is from controlling the epidemic and yet one of the targets of sustainable development goal (SDG) 3 is to end the HIV/AIDS epidemic by 2030 [2]. In order to achieve this ambitious target, the global community has embarked on promoting universal health coverage such as early provision of antiretroviral treatment (ART) across all populations including adolescents aged 10 - 19 years living with HIV (ALHIV), irrespective of clinical or immunological status [3]. Although this recommendation has been adopted by several countries including Uganda, many studies have reported lower rates of ART adherence and viral load (VL) suppression among the ALHIV [3] [4] [5] [6] [7] than other subpopulations. VL suppression is an important indicator of good HIV treatment response and curtails transmission which is a key factor in the AIDS epidemic control. Non-VL suppression is associated with poor clinical outcomes and could explain why AIDS related deaths have not improved among the ALHIV especially in sub-Saharan Africa [8] and yet AIDS related mortality has reduced by almost $50 \%$ in the last 15 years in the general population [9].

Uganda's Ministry of Health $(\mathrm{MoH})$ adopted the World Health Organization (WHO)'s recommendation to use VL monitoring as the most accurate measure of ART response among all people living with HIV (PLHIV) [10] [11]. The 2016 consolidated national ART guidelines recommend 6-monthly VL monitoring among the children and adolescents living with HIV given the fact that this population has a higher prevalence of non-adherence and poor VL suppression (VLS) [10]. Indeed the 2017 Uganda population based HIV Impact Assessment survey (UPHIA) reported a national VLS rate of 39\% among children and adolescents below 15 years of age [12]. The available evidence highlights several factors that have contributed to low VLS and adherence among the adolescents. These include stigma, side effects of drugs and laxity to treatment especially among perinatally infected ALHIV, non-disclosure, male adolescent aged 15 - 19 and inadequate family support [4] [5] [13] [14]. Some of these factors could be attributed to the fact that adolescents face many neurodevelopmental, psychosocial and economic challenges as they grow [6] [7] [15]. In light of this, we found that the VLS rate among the ALHIV at The AIDS Support Organization (TASO) Mbale was $63.1 \%$ in quarter four of 2016. This was unacceptably much lower 
than the $90 \%$ target set by UNAIDS in 2014 . Therefore, we proposed a quality improvement approach to address this problem and improve viral load suppression among the ALHIV from $63.1 \%$ to $90 \%$ between quarter 4 of 2016 to quarter 4 of 2017.

Quality improvement (QI), the science of identifying gaps in health service and providing practical solutions to close them, is documented as a simple, practical and cost-effective approach in meeting or surpassing required standards of care. The Uganda ministry of health highly recommends application of quality improvement approach to address VL challenges in HIV care [11]. This is because of the documented successes in its use to improve quality of health care in various key areas of services provision. For example, a study in northern Uganda reported improved Tuberculosis (TB) case notification from 171/100,000 to 223/100,000 using continuous quality improvement (CQI) approach to care [16]. Another study from midwestern Uganda applied the same approach and improved retention of ALHIV in care from $29.3 \%$ to $100 \%$ within 12 months [8]. In addition, Leighann E Kimble et al. also documented the use of QI approach in addressing hospital acquired infections and antimicrobial resistance [17]. Therefore, our hypothesis was that quality improvement approach would increase the VLS rate among the ALHIV from $63.1 \%$ to $90 \%$ within 12 months.

The consequences of VL non-suppression are well documented. They include poor quality of life, development of drug resistance and transmission of new infections [11]. Despite these, well documented successful interventions to address VL challenges among the ALHIV are very limited. Therefore, it is imperative that successful endeavors are well documented and shared. So, this paper demonstrates successful interventions that helped TASO Mbale achieve at least 90\% VLS rate among the adolescents living with HIV from January 2017 to December 2017. This will complement the existing body of evidence to form a stronger ground for future policy and program guidance.

\section{Methodology}

\subsection{Study Design}

We implemented a quality improvement approach to correct the gaps of low VLS among the ALHIV at TASO Mbale. QI uses basic principles which include routine data use, client focus, strong leadership, focusing on systems and processes, and team building to correct gaps in health care provision [18]. Innovative approaches were employed to close the inadequacies.

\subsection{Site Setting}

TASO Mbale is one of the 11 centers of Excellence (CoE) of TASO Uganda [19]. It is located within Mbale regional referral Hospital in Mbale Municipality, about $230 \mathrm{Kms}$ from Kampala capital City. The CoE operates within a radius of $75 \mathrm{kms}$, including the districts of Mbale, Sironko, Bududa, Namisindwa, Manafwa, Budaka, Kibuku, Pallisa and Butebo. TASO Mbale, a special HIV only 
clinic, offers comprehensive HIV/TB prevention and treatment services such as safe male circumcision, HIV Testing Services, care and treatment. By the end of 2016 the CoE had an active clientele of 6041 of whom 440 (300 females and 140 males) were adolescents aged $10-19$. Of the 440 adolescents, 387 were on first line ART regimens and 53 were on second line ART drugs.

The center operates throughout the week with clinic days on Tuesdays and Thursdays. Adults are separated from the adolescents whose are reviewed once a month. Usually, a team is selected to run the adolescent clinic on a monthly basis. The model of the Adolescent HIV clinic at TASO Mbale is an independent one [20]. QI has been routinely implemented at the facility spearheaded by the QI focal person.

\subsection{The QI Steps Employed}

\section{Step 1 (Problem Identification)}

Using program data from the Uganda national viral load dashboard, we found that at TASO Mbale CoE, the VLS rate among the adult clients (aged 20 years and above) was $85.7 \%$ while that among the adolescents was $63.1 \%$ in the fourth quarter of 2016 [21]. So, consequently, the Center QI committee decided to address this huge gap using a QI approach. The committee started by dedicating half a day to build QI capacity of other health workers at the CoE. This was done through a Continuous Medical Education (CME) where the committee shared basic QI facts such as principles of QI, dimensions of QI, problem identification and analysis tools and use of data in QI. We also used practical examples through simulations to drive home critical areas of QI. Finally, a dedicated team was selected to undertake this project given the importance of adolescent HIV care in driving public health epidemic control both nationally and internationally.

\section{Step 2 (Root Cause Analysis)}

We combined both brainstorming and fishbone methods (Figure 1) [22] to perform this operation. Using the 5-whys tool, we analyzed each identified problem by asking why, five times till a logical solution believed to be a root cause of low VLS among the ALHIV was arrived at. We then realized two main categories of root causes: adolescent related and health facility related ones.

Adolescent related challenges were: missing clinic appointment and poor adherence while health facility related challenges include disorganized ALHIV clinic set up, inadequate follow-up and low data use for informed decision making. TASO Mbale CoE adolescent HIV clinic was run by random and non-dedicated group of health workers with poor coordination. This once a month clinic would see more than 100 adolescents swarm the adolescent service area every second Wednesday of the month. In addition, there was absence of a responsible officer to track appointment of the ALHIV and initiate follow up in case of need. Finally, data use for informed decision making was restricted to the Monitoring and Evaluation team which minimized quality service provision. 


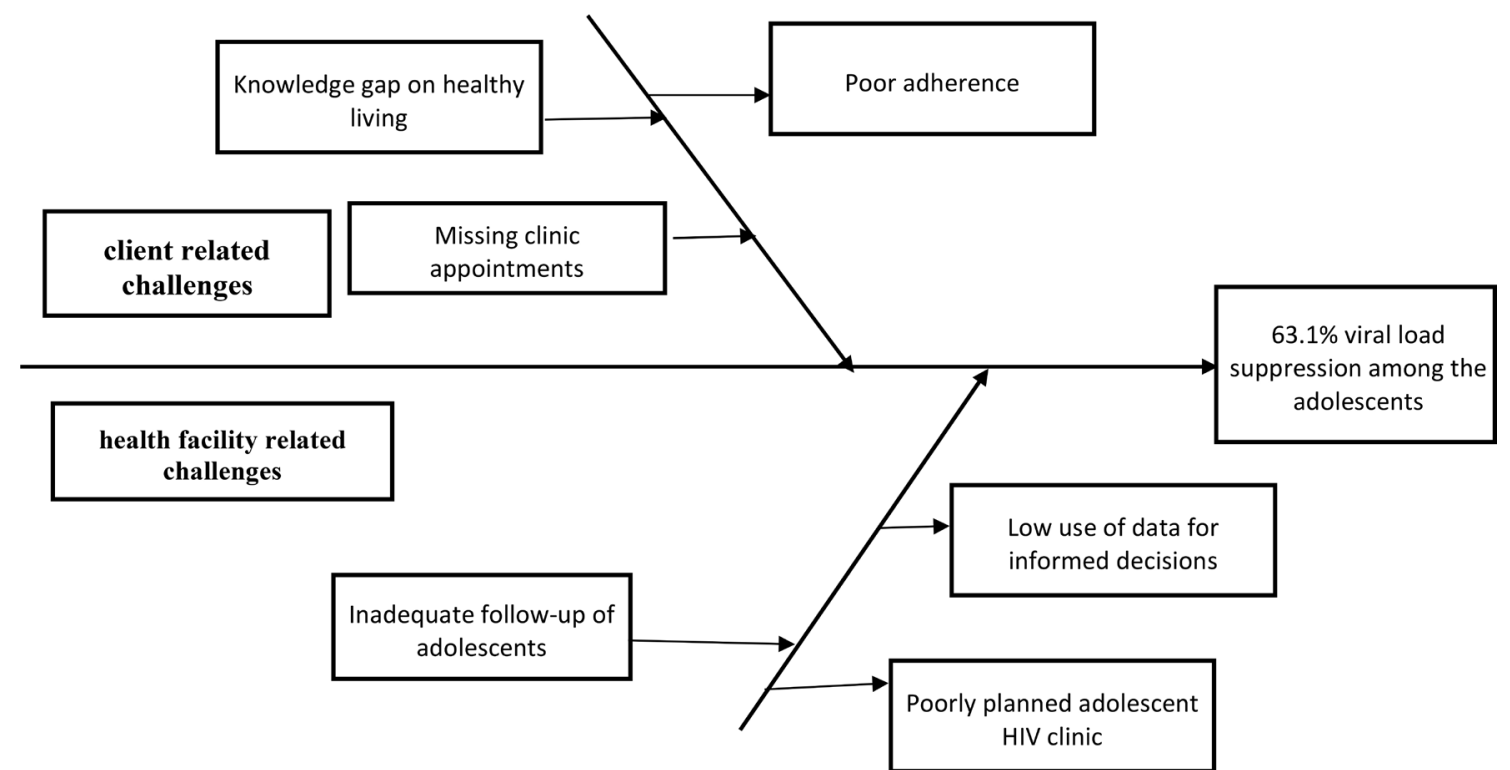

Figure 1. A fishbone diagram with the root causes of low viral load suppression related to the adolescents and health facility.

\section{Deliberating on Solutions}

To address the problem of disorganization of the adolescent HIV clinic, a dedicated and committed team was formed. This team included a clinician, a counsellor and adolescent peers. In addition, the adolescent clinic was re-organized into multiple times (twice in a week) run on the same days like the children and adult clinics. This was then followed by redistributing the clients favorably to the different clinic days, in manageable numbers not exceeding 20 per clinic day.

Regarding the inadequate follow-up of the adolescents who miss viral load bleeding, an appointment register was introduced to track and monitor appointment behavior of the ALHIV. This register would capture client details such as registration numbers, names, age and contact information. Another locally made register was also used to track viral load coverage and results. The counsellor was responsible for the appointment register while the clinician took charge of the viral load register. A list of the adolescents who were lost or missed appointment and those due for VL screening was generated by the Monitoring and Evaluation team for follow-up. The peers provided routine health education covering a wide range of topics including positive living and viral load monitoring. Both group and individual counselling were provided to address general and unique challenges to quality adherence to medication among the ALHIV. During group sessions, adolescents with suppressed viral load (VL $<1000$ copies/mL) shared experiences with their non-suppressing counter parts (clients with $\mathrm{VL} \geq 1000$ copies/mL).

Finally, both phone calls and home visits were used to fast-track follow-up of the ALHIV who did not attend clinic within scheduled time. While the Uganda Ministry of health defines missed appointment as clients who do not attend clinic visits with 7 days of their scheduled appointment [9], we made follow-ups 
at the end of each clinic day for those with available functional telephone contacts. Physical visits were done for those adolescents who consented for home visit during registration.

\section{Implementing the Changes}

We implemented The Plan-Do-Study-Act (PDSA) cycle, a set of steps in implementation science that helps in observing and documenting tested changes [23] [24]. This approach helped us monitor and evaluate the tested changes implemented to improve viral load suppression among the ALHIV at TASO Mbale. During our QI implementation, we tested the following changes: firstly, committing a dedicated team; secondly, increasing the number of adolescent clinic days and integrating them on the same day as the ones for adults and the children; lastly redistributing the ALHIV equitably into the different clinic days. These implementations took place in January and February 2017. In the second phase, the Monitoring and Evaluation team generated a list of ALHIV who were lost and those due for VL screening for accelerated follow-up. Subsequently, this would be done by using the available registers (appointment and Viral Load registers). The dedicated team provided continuous, comprehensive HIV care and treatment support services including family planning and Gender Based Violence to ALHIV at the CoE. In the third phase, we re-categorized the adolescents into two age-based strata. The adolescents aged 10 - 14 years were provided with clinical reviews during Tuesday clinic hours while those aged 16 - 19 years were offered services during the Thursday clinic hours. Finally, we conducted one adolescent-guardian-health worker in the first half of 2017 to discuss multiple issues affecting the ALHIV receiving services from our center.

\subsection{Statistical Analysis and Handling of the Results of the Project}

Viral load suppression defined as suppressed when the result was less than 1000 copies/mL and non-suppressed if $\geq 1000$ copies/mL as defined by the $\mathrm{MoH}$ [10] [11]. The data was generated from the national VL dashboard. VLS was calculated by dividing the number of adolescents who suppressed their VL by the total number of valid samples tested during the quarter. The findings were documented in a QI journal that has the project objective, problem statement of the project, indicators for the objective, planned and tested changes, lessons learnt documented and line graph was updated on a quarterly basis. The center team was provided with progress updates on a monthly basis.

\section{Results}

\begin{tabular}{cccccc}
\hline Period (in quarters) & $\begin{array}{c}\text { Quarter 4 } \\
\text { 2016 }\end{array}$ & $\begin{array}{c}\text { Quarter 1 } \\
\text { 2017 }\end{array}$ & $\begin{array}{c}\text { Quarter 2 } \\
\mathbf{2 0 1 7}\end{array}$ & $\begin{array}{c}\text { Quarter 3 } \\
2017\end{array}$ & $\begin{array}{c}\text { Quarter 4 } \\
2017\end{array}$ \\
\hline $\begin{array}{c}\text { Number of clients with } \\
\text { suppressed VL results }\end{array}$ & 130 & 139 & 223 & 240 & 148 \\
Valid samples tested for VL & 206 & 218 & 255 & 246 & 162 \\
VLS rate & $63.10 \%$ & $63.80 \%$ & $87.50 \%$ & $97.60 \%$ & $91.40 \%$ \\
\hline
\end{tabular}




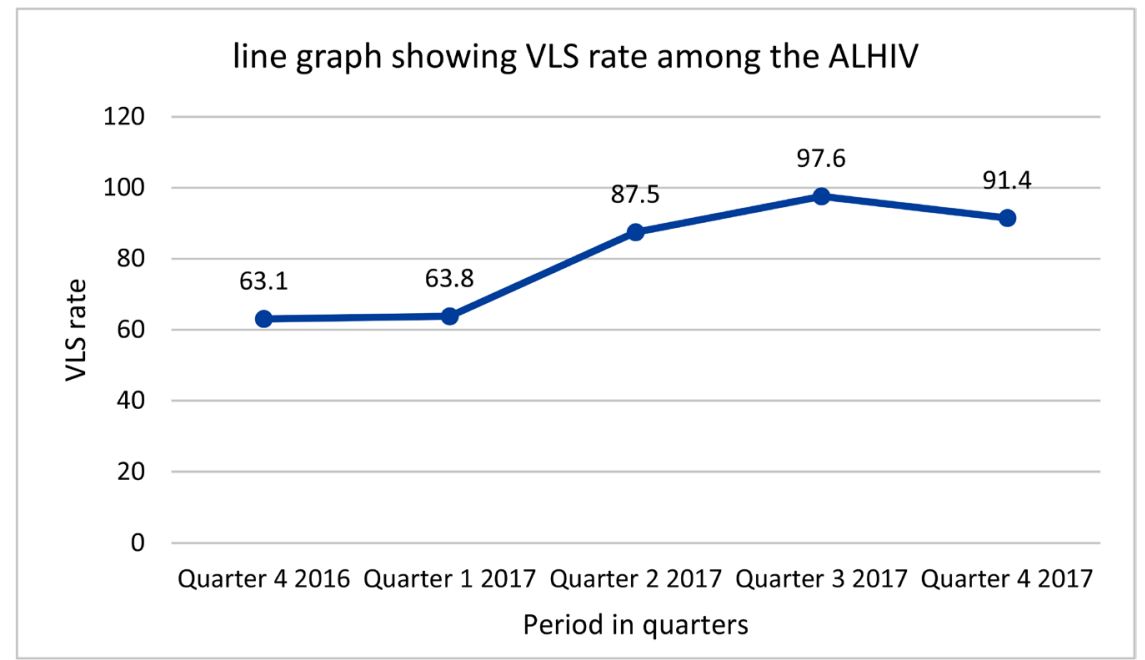

Figure 2. The trend in VLS rate among the ALHIV at TASO Mbale over time.

Before the QI interventions, VLS among the ALHIV at TASO Mbale was at 63.1\% (130/206) in the fourth quarter of 2016 (October to December) from Figure 2. However, following the start of the implementation of the QI tested changes, VLS rose slightly from $63.1 \%$ to $63.8 \%(139 / 218)$ in the first quarter of 2017. This picture continued to improve in Quarter 2 of 2017 with a VLS rate of 87.5\% (223/246), 97.6\% (240/246) in Quarter 3 and 91.4\% (148/162) in the fourth quarter of 2017.

\section{Discussions}

The public health benefit of sustained viral load suppression among the ALHIV cannot be over-emphasized. Considering the low uptake of HIV prevention services among the adolescents and young people [9], achieving sustained Viral load suppression may yet play an important role in reducing HIV new infections in this sub-population, potentially contributing to 6 sustainable development goals [25]. Our findings demonstrate that by applying simple, practical and cost-effective interventions, it is possible to achieve a remarkably high VLS among the ALHIV. The aim of this QI project was to improve viral load suppression among ALHIV from 63.1\% in quarter 42016 to 90\% in quarter 4 of 2017. Uganda was among the first countries in Africa to adopt the application of Quality improvement approaches in health care service deliveries in early 1990s [18]. The ministry of health now emphasizes its application at all levels of health service delivery as a cost-effective approach for closing gaps in care including viral load suppression [10] [11]. One of the objectives of the Uganda national 2015-2020 Health Sector QI framework and strategic plan is strengthen the framework for documentation, reporting and sharing of QI projects [26]. Lastly, quality care is intimately embedded in all the WHO six building blocks of health systems (health service delivery, health workforce, essential medicines, Health Information, Health Financing, and Leadership and Governance) [8] [27].

We achieved our objective by reaching a VLS rate of $97.6 \%$ in quarter 3 of 
2017 and $91.4 \%$ in quarter 4 . The introduction of the appointment register in the clinic was instrumental in fast-tracking follow-up of clients who missed their scheduled appointment. The clinic team analyzed and summarized appointment behavior of ALHIV at the end of each clinic day. This helped us identify those who had missed so that follow-up would start immediately for those with reliable telephone contacts. In addition, making use of a list of eligible clients for viral load screening on appointment improved viral load coverage in this age group. Those who were successfully followed up were re-scheduled to another clinic visit of convenience. The successful use of appointment register to track missed appointments has also beenreported by another study in mid-Western Uganda in improving retention rate among ALHIV in care [8]. Therefore, a robust monitoring and evaluation is a key factor in a successful quality health care program. Home visits were used for those clients who had consented to it. This activity was particularly important in addressing both family and community related challenges including gender-based violence among the ALHIV and/or their mothers. So, home visit teams (usually involved adolescent peers, lay health workers, a counsellor and/or clinician) provided HIV services in integrated manner including mobilizing communities to provide social protection to the adolescents.

TASO Mbale CoE, being a counselling organization placed particular emphasis on addressing psychosocial issues among the adolescents in general and those with non-suppressed viral loads particularly. Both individual and group sessions were implemented. During group sessions, the adolescents were often mixed up (both those with viral load suppressed status and the non-suppressed counter-parts) while provided an opportunity for sharing experiences. A wide range of positive living topics were also covered during the group sessions such as sexual and reproductive behavior, adherence to medication, viral load suppression and HIV prevention. The clients were particularly motivated with the fact that a virally suppressed client is unlikely to transmit HIV to a sexual partner. Individual counselling was prioritized among the virally non-suppressed individuals. Several studies have reported the importance of enhanced adherence support following viral load non-suppression. For example, a Zimbabwean study reported a significant level of suppression among the adolescents (34\%) following enhanced adherence counselling after an initial non-suppressed result [28]. Another study in South-Africa also concluded that viral load suppression was associated with effective adherence counselling [29] although a study in Uganda reported low suppression rates among children and adolescents following intensive adherence counselling [30]. However, this particular study used routine program data and could not confirm the quality of the counselling provided to the children and the adolescents.

One of the most significant interventions was increasing the number of interactions with the ALHIV through creating more clinic days to match those for adults and children. This is one of the models of care among the ALHIV [31]. During our planning phase, we agreed to redistribute the adolescents such that 
manageable numbers would be reviewed every Tuesday and Thursday, like the adults and children. This approach mitigated several challenges. First, the adolescents enjoyed high quality care because of smaller manageable numbers of about 20 per clinic day and a dedicated team that understood them well. Before the intervention, more than 100 ADLHIV would be reviewed by a randomly selected team. As it is a common practice, what belongs to everyone, belongs to nobody. The adolescents were then prone to receiving below standard services. Secondly, it motivated some parents to accompany their adolescents especially those who were receiving ART service from TASO Mbale due to synchronization of the appointment schedules, hence improving appointment adherence. Lastly, both the clients and clinic teams bond well, reducing stigma that inherently exists in the setting of HIV care. Due to observed high prevalence of pregnancy among the adolescents in our care during the first half of 2017, we regrouped the adolescents with younger ones (10-14) reviewed on Tuesdays and older ones on Thursdays. Remarkably, the incident of pregnancy had drastically reduced by December 2017. Our hypothesis was that, the older adolescents were influencing the younger ones negatively into engaging in risky sexual behavior. However, this evidence is low quality due to lack of a control group. The intervention may not be feasible in clinics with small adolescent populations and those with low human resources for health. In a separate analysis, these interventions also facilitated smooth transition of the eligible youth (aged 20 years and above) from the adolescent clinic to that of adults. We transitioned 50 out of 61 eligible youth who were in the adolescent clinic between July 2017 and December 2017.

Finally, we organized and conducted one meeting among the ALHIV, some selected teachers, guardians, parents and the health workers at TASO Mbale in the first half of 2017. During this one-day meeting/workshop, we shared common and unique challenges facing our adolescent clients in care and sought probable solutions together with all the stakeholders. This improved relationships between the adolescents and their guardians and also the teachers, reducing stigma and discrimination, improving adherence to medication. We believe this intervention played a key role in achieving better virologic outcomes in the second half of 2017.

\section{Study Limitations}

Our study applied practical and cost-effective interventions to improve viral load suppression among the adolescents living with HIV. These findings are a powerful display of the effectiveness of quality improvement interventions in closing gaps that exist in health care service delivery at all levels. However, it is worth noting that, most health workers have not familiarized themselves with the concept of quality improvement. In addition, we believe these strategies can be highly successful in similar settings.

This study however had many limitations: 
- Lack of key demographic information among the adolescents such as age and sex.

- There was a general lack of a control group.

- We documented evidence from one site which may not have considered other fundamental factors that exist in certain unique settings.

Regardless, this document provides specific insightful approaches in reaching the HIV epidemic control by achieving viral load suppression among the ALHIV, who are the epicenter of the AIDS epidemic.

\section{Conclusion}

1) The use of quality improvement in mitigating gaps in HIV service delivery is highly effective and efficient.

2) A dedicated clinical team to handle the adolescent HIV care is essential in ensuring provision of adolescent friendly services.

3) Improving monitoring and evaluation is key. With the introduction of registers and other tracking methods, we realized excellent outcomes in both keeping appointments, timely viral load bleeding/screening and suppression. We therefore implore health care providers to employ these interventions especially those in centers of excellence.

\section{Author Contributions}

All the authors listed made substantial contribution to the conception, design of the work, analysis and interpretation of findings. They all participated in drafting and critically making important intellectual contribution. We have all approved the final version and agree to be fully accountable for the work in case of questions related to integrity or accuracy arise.

\section{Acknowledgements}

TASO Mbale QI committee for capacity building. We appreciate other clinic team members especially Godfrey Wasike, Andrew Kisame, Francis Madoi, Merina Asasira, Twaha Mafabi Nangoli and Catherine Achola for their dedication to serving the adolescent clients. We acknowledge USAID RHITES-E for supporting the operations of TSO Mbale both financially and technically.

Finally, we highly appreciate Mr. Sebastian Jalameso of Uganda National Curriculum Development Center for editing this work.

\section{Conflicts of Interest}

The authors declare no conflicts of interest regarding the publication of this paper.

\section{References}

[1] Avert: Global Information and Education on HIV and AIDS. https://www.avert.org/global-hiv-and-aids-statistics 
[2] Johnston, R.B. (2016) Arsenic and the 2030 Agenda for Sustainable Development. Arsenic Research and Global Sustainability-Proceedings of the 6th International Congress on Arsenic in the Environment, New York, 25-27 September 2015, 12-14.

[3] World Health Organization (2016) Consolidated Guidelines on the Use of Antiretroviral Drugs for Treating and Preventing HIV Infection: Recommendations for a Public Health Approach.

[4] Inzaule, S.C., Hamers, R.L., Kityo, C., Rinke De Wit, T.F. and Roura, M. (2016) Long-Term Antiretroviral Treatment Adherence in HIV-Infected Adolescents and Adults in Uganda: A Qualitative Study. PLoS ONE, 11, e0167492. https://doi.org/10.1371/journal.pone.0167492

[5] Nabukeera-Barungi, N., Elyanu, P., Asire, B., Katureebe, C., Lukabwe, I., Namusoke, E., Musinguzi, J., Atuyambe, L. and Tumwesigye, N. (2015) Adherence to Antiretroviral Therapy and Retention in Care for Adolescents Living with HIV from 10 Districts in Uganda. BMC Infectious Diseases, 15, 520. https://doi.org/10.1186/s12879-015-1265-5

[6] Zanoni, B.C., Sibaya, T., Cairns, C., Lammert, S. and Haberer, E. (2017) Higher Retention and Viral Suppression with Adolescent-Focused HIV Clinic in South Africa. PLoS ONE, 12, e0190260. https://doi.org/10.1371/journal.pone.0190260

[7] Cluver, L.D., Toska, E., Orkin, F.M., Meinck, F., Hodes, R. and Yakubovich, A.R. (2016) Achieving Equity in HIV-Treatment Outcomes: Can Social Protection Improve Adolescent ART-Adherence in South Africa? AIDS Care, 28, 73-82. https://doi.org/10.1080/09540121.2016.1179008

[8] Izudi, J., Mugenyi, J., Mugabekazi, M., Muwanika, B., Tumukunde Spector, V., Katawera, A. and Kekitiinwa, A. (2018) Retention of HIV-Positive Adolescents in Care: A Quality Improvement Intervention in Mid-Western Uganda. BioMed Research International, 2018, Article ID: 1524016. https://doi.org/10.1155/2018/1524016

[9] Govender, K., Masebo, W.G.B., Nyamaruze, P., Cowden, R.G., Schunter, B.T. and Bains, A. (2018) HIV Prevention in Adolescents and Young People in the Eastern and Southern African Region: A Review of Key Challenges Impeding Actions for an Effective Response. The Open AIDS Journal, 12, 53-67. https://doi.org/10.2174/1874613601812010053

[10] Jagwe, J., Kamya, M., et al. (2016) Consolidated Guidelines for Prevention. Kampala.

[11] MoHCC (2013) Consolidated Guidelines on the Prevention and Treatment of HIV. Kampala.

[12] (2019) Uganda Population-Based HIV Impact Assessment, UPHIA 2016-2017. $1-252$.

[13] Chhim, K., Mburu, G., Tuot, S., Sopha, R., Khol, V., Chhoun, P. and Yi, S. (2018) Factors Associated with Viral Non-Suppression among Adolescents Living with HIV in Cambodia: A Cross-Sectional Study. AIDS Research and Therapy, 15, 20. https://doi.org/10.1186/s12981-018-0205-Z

[14] Jobanputra, K., Parker, L.A., Azih, C., Okello, V., Maphalala, G., Kershberger, B., Khogali, M., Lujan, J., Antierens, A., Teck, R., Ellman, T., Kosgei, R. and Reid, T. (2015) Factors Associated with Virological Failure and Suppression after Enhanced Adherence Counselling, in Children, Adolescents and Adults on Antiretroviral Therapy for HIV in Swaziland. PLoS ONE, 10, e0116144. https://doi.org/10.1371/journal.pone.0116144

[15] WHO (2019) Adolescent-Friendly Health Services for Adolescents Living with HIV: 
From Theory to Practice.

[16] Karamagi, E., Sensalire, S., Muhire, M., Kisamba, H., Byabagambi, J., Rahimzai, M., Mugabe, F., George, U., Calnan, J., Seyoum, D. and Birabwa, E. (2018) Improving TB Case Notification in Northern Uganda: Evidence of a Quality Improvement-Guided Active Case Finding Intervention. BMC Health Services Research, 18, Article No. 954. https://doi.org/10.1186/s12913-018-3786-2

[17] Kimble, L.E., Massoud, M.R., et al. (2017) Using Quality Improvement to Address Hospital-Acquired Infections and Antimicrobial Resistance.

[18] Ministry of Health of Uganda (2015) The Quality Improvement Methods: A Manual for Health Workers in Uganda. 1-64.

[19] TASO (2018) TASO Strategic Plan 2018-2022.

[20] Asire, B., Nabukeera-Barungi, N., Elyanu, P., Katureebe, C., Lukabwe, I., Namusoke, E., Musinguzi, J., Tumwesigye, N. and Atuyambe, L. (2017) Adolescent HIV Care and Treatment in Uganda: Care Models, Best Practices and Innovations to Improve Services. Journal of Infectious Diseases \& Preventive Medicine, 5, 1-6. https://doi.org/10.4172/2329-8731.100015

[21] Central Public Health Laboratory: Ugnda National viral load Dashboard. https://vldash.cphluganda.org

[22] Fishbone Diagram.

https://www.qimacros.com/fishbone-diagram-template/ishikawa-fishbone-customi zation

[23] Paul, W. and Ben, G. (2004) Completing the Circle: From PD to PDSA. International Journal of Health Care Quality Assurance, 17, 349-358. https://doi.org/10.1108/09526860410557606

[24] Institute for Helthcare Improvement: Science of Improvement: How to Improve. http://www.ihi.org/resources/Pages/HowtoImprove/ScienceofImprovementHowtoI mprove.aspx

[25] UNAIDS (2020) HIV Prevention 2020 Road Map: Accelerating HIV Prevention to Reduce New Infections by $75 \%$.

[26] MOH-Uganda (2015) Health Sector Quality Improvement Framework and Strategic Plan 2015/16-2019/20.

[27] WHO (2010) Monitoring the Building Blocks of Health Systems: A Handbook of Indicators and their measurement strategies. 110 (2010).

https://www.who.int/workforcealliance/knowledge/toolkit/26/en/

[28] Bvochora, T., Satyanarayana, S., Takarinda, K.C., Bara, H., Chonzi, P., Komtenza, B., Duri, C. and Apollo, T. (2019) Enhanced Adherence Counselling and Viral Load Suppression in HIV Seropositive Patients with an Initial High Viral Load in Harare, Zimbabwe: Operational Issues. PLoS ONE, 14, e0211326.

https://doi.org/10.1371/journal.pone.0211326

[29] Fox, M.P., Berhanu, R., Steegen, K., Firnhaber, C., Ive, P., Spencer, D., Mashamaite, S., Sheik, S., Jonker, I., Howell, P., Long, L. and Evans, D. (2016) Intensive Adherence Counselling for HIV-Infected Individuals Failing Second-Line Antiretroviral Therapy in Johannesburg, South Africa. Tropical Medicine \& International Health, 21, 1131-1137. https://doi.org/10.1111/tmi.12741

[30] Nasuuna, E., Kigozi, J., Babirye, L., Muganzi, A., Sewankambo, N.K. and Nakanjako, D. (2018) Low HIV Viral Suppression Rates Following the Intensive Adherence Counseling (IAC) Program for Children and Adolescents with Viral Failure in Public Health Facilities in Uganda. BMC Public Health, 18, 1048. 
https://doi.org/10.1186/s12889-018-5964-X

[31] Asire, B., et al. (2017) Adolescent HIV Care and Treatment in Uganda: Care Models, Best Practices and Innovations to Improve Services. Journal of Infectious Diseases \& Preventive Medicine, 5, Article ID: 1000150.

https://doi.org/10.4172/2329-8731.1000150 